Rloes Revue internationale de pédagogie de l'enseignement supérieur

26(1) | 2010

Varia - printemps 2010

\title{
Le projet professionnel et personnel des étudiants, assise de leur professionnalisation?
}

Daniel Bart et Michel Fournet

\section{OpenEdition}

Édition électronique

URL : http://journals.openedition.org/ripes/314

DOI : 10.4000/ripes.314

ISSN : 2076-8427

Éditeur

Association internationale de pédagogie universitaire

Référence électronique

Daniel Bart et Michel Fournet, « Le projet professionnel et personnel des étudiants, assise de leur professionnalisation? », Revue internationale de pédagogie de l'enseignement supérieur [En ligne], 26(1) | 2010, mis en ligne le 10 mai 2010, consulté le 07 septembre 2020. URL : http:// journals.openedition.org/ripes/314; DOI : https://doi.org/10.4000/ripes.314

Ce document a été généré automatiquement le 7 septembre 2020.

Article L.111-1 du Code de la propriété intellectuelle. 


\title{
Le projet professionnel et personnel des étudiants, assise de leur professionnalisation?
}

\author{
Daniel Bart et Michel Fournet
}

1 Si d'aucuns considèrent aujourd'hui la pensée et les pratiques dites «à projets » comme l'essence du capitalisme moderne (Boltanski \& Chiapello, 1999), l'usage du projet n'est pas chose nouvelle en éducation (Dewey, 1938). Figure globale de la vie sociale, «surface de projection des aspirations des individus et référence obligée des organisations » (Boutinet, 1986, p. 17), le projet est en effet considéré comme un mode privilégié d'adaptation dans des contextes incertains, notamment de formation ou d'emploi. Dans l'enseignement supérieur français, les premières expérimentations de dispositifs pédagogiques de type projet datent du milieu des années 1980 alors que les travaux québécois fondateurs de cette approche datent des années 1970 (Pelletier, Noiseux \& Bujold, 1974).

2 Mais c'est au cours des années 2000 que les unités d'enseignement «Projet professionnel » supposées diminuer les risques d'échec dans les premières années des formations universitaires généralistes se sont multipliées en France (Mérini \& Séré, 2001). De même, les diplômes universitaires de technologie comportent depuis 2005 un module «Projet professionnel et personnel » conçu comme un moyen d'accroître la qualité d'insertion de leurs diplômés. L'essor du recours aux activités pédagogiques à projets à l'université peut ainsi être mis en relation avec la seconde ${ }^{1}$ massification universitaire des années 1980 et le renforcement plus récent de la professionnalisation des formations supérieures. Le lien entre les projets de jeunes en formation et les emplois qu'ils ont occupés ultérieurement a d'ailleurs pu être analysé (Beret, 2002), de même que l'efficacité des démarches d'accompagnement pédagogique des étudiants sur la formalisation de leur projet professionnel (Masclet \& Leconte, 2007).

Dans cet article, nous nous intéressons plutôt aux conditions d'émergence et à la structuration de tels projets dans le cas d'un diplôme professionnel universitaire de niveau plus élevé (Master, second cycle universitaire). Notre recherche vise à mieux 
comprendre comment les activités pédagogiques à projets à l'université sont susceptibles de favoriser un développement professionnel chez des étudiants d'un relativement haut niveau de formation. En cherchant à dégager empiriquement l'agencement de projets de ces étudiants, notre objectif est d'analyser en quoi ces projets peuvent soutenir la pluralité des engagements et des apprentissages de ces derniers. Dans cette perspective, les projets personnels et professionnels seront étudiés d'après les travaux d'Ardoino (1987), à travers leurs deux composantes porteuses de complémentarité et d'opposition: d'une part, les aspirations, les visées de l'étudiant, faites de valeurs, de référents socioculturels ou professionnels et d'autre part, l'ébauche, la trame constituant la traduction opératoire de telles intentions. Les figures du projet sont multiples et si nous nous focalisons dans cette recherche sur un projet d'insertion professionnelle d'étudiants inscrit dans un cadre plus général d'attentes et de représentations constitutif de leur projet personnel, nous considérons également que ceux-ci ne peuvent être déconnectés du projet institutionnel de formation du Master (Roegiers, 2003).

Cette approche théorique sera précisée après avoir présenté le contexte de la recherche. Nous exposerons ensuite notre méthodologie d'investigation avant d'analyser les projets des étudiants et la manière dont ces derniers "traduisent » le programme de la formation en appui "stratégique» à leur développement professionnel. En conclusion, nous montrerons qu'au-delà des recours systématiques et instrumentaux aux projets en formation supérieure, ces travaux appellent des régulations et aménagements pédagogiques à même de tenir compte de la configuration des projets mise en évidence et d'appuyer leur rôle de support au processus de professionnalisation.

\section{Le contexte de la recherche}

Notre recherche porte sur les projets d'une cohorte d'une vingtaine d'étudiants inscrits dans une formation universitaire professionnelle en 2007-2008: le Master "Accompagnement et conseil en développement professionnel» organisé par l'Université de Toulouse II (France). Ce diplôme vise à former les étudiants aux pratiques et dispositifs de conseil et d'accompagnement dans les champs de l'éducation, du travail social ou du recrutement. Il se prépare en un an à la suite de quatre années d'études supérieures en Sciences Humaines et Sociales ou d'une admission par validation des acquis de l'expérience. Comme tous les Masters de l'enseignement supérieur français, il conduit donc à un niveau de formation de cinq années post baccalauréat.

Le référentiel de la formation précise que les enseignements dispensés visent l'acquisition de connaissances relatives aux théories du conseil, de l'évaluation et de l'accompagnement et le développement de compétences en matière d'analyse des pratiques professionnelles, de conduite d'entretien, de réalisation d'audit, de conception de dispositifs de formation et de production d'écrits professionnels (rapports, synthèses...). Cette formation s'appuie sur la réalisation de 320 heures de stage en entreprise ou en institution publique durant l'année d'étude (cabinets de conseil, centres de formation ou d'insertion, services des ressources humaines).

Outre l'alternance entre l'université et le terrain de stage, ce type de parcours professionnalisant se distingue des formations généralistes par l'importance de la 
référence au projet professionnel des étudiants. En effet, le projet de l'étudiant et sa pertinence servent à la fois de critère de sélection d'entrée en formation, de guide pour les choix des stages et de repères pour l'élaboration des thématiques de mémoire professionnel. De plus, certaines séquences pédagogiques visent à accompagner individuellement et collectivement les stagiaires dans la formalisation et la mise en œuvre de leur projet. Selon Beckers (2007, p. 243), cette approche pédagogique par projet aurait, comme situation d'intégration, trois effets positifs pour les étudiants : " (des effets) motivationnels (accrocher, susciter l'engagement des étudiants et leur persévérance face aux tâches scolaires), cognitifs (acquérir des savoirs et des démarches mobilisables parce que construits par le sujet en réponse à des besoins éprouvés dans l'interaction avec l'environnement) et socio-affectifs (apprendre à assumer des responsabilités, collaborer, dynamiser autrui...).»

Mais l'enjeu heuristique et pédagogique d'une meilleure connaissance du processus d'élaboration et de structuration des projets se pose avec d'autant plus d'acuité que les participants à ce diplôme se préparent à exercer des activités dans un secteur aux contours incertains et où les capacités d'adaptation, d'initiative et de polyvalence sont centrales (Bart,sous presse). Ces futurs professionnels doivent être capables d'élaborer et de mettre en œuvre des démarches d'accompagnement et de conseil tant dans les champs de l'orientation et du recrutement (bilan de compétences, gestion des ressources humaines,...) que dans ceux du conseil en formation et du suivi de publics particuliers (actions d'insertion, de reconversion,...). De plus, les métiers de l'accompagnement et du conseil font prévaloir une logique d'offre proactive -celle des diplômés- à une logique d'anticipation de la demande -celle d'employeurs potentiels.

Stratège créatif en amont, gestionnaire réaliste en cours et négociateur habile en fin de formation, réflexif tout au long, tel apparaît l'idéal type de l'étudiant de ce Master. «Produire sa vie» écrivent Pineau et Michèle (1983), «assumer sa professionnalisation" proposons-nous plus modestement. C'est dire que nous considérons la formation de Master comme un "moment", avec ou sans l'idée de brièveté, mais surtout comme condition d'un mouvement ${ }^{2}$. Dans la vie des sujets, ce moment ponctue la trajectoire biographique sensible aux déterminations des origines et ouvre, potentiellement, sur un parcours attendu comme moins sensible aux déterminismes, un espace de possibles où se trame un projet global, tout à la fois professionnel et personnel.

\section{Projet professionnel et personnel de l'étudiant et projet institutionnel de formation}

10 Il est commun de décomposer l'activité complexe de projet en trois phases, avec une période initiale de créativité, un engagement fortifié progressivement par des choix décisifs et une réalisation placée pas à pas sous le regard d'autrui (Boutinet, 1990). Il convient cependant de souligner que la constitution et l'agencement du projet, par essence singulier, évoluent, se ramifient et se spécifient en déclinaisons mineures, complémentaires, substitutives ou compensatoires, sans linéarité stricte. Difficile en effet, quand l'improvisation s'impose face à la nouveauté des situations de formation de séparer clairement la conception et la réalisation, de passer avec certitude du dessein au dessin, de fixer une perspective nette. L'expérience personnelle offre alors quelques repères, d'autant mieux qu'elle est professionnelle bien sûr. L'élaboration d'un projet 
professionnel et personnel n'est donc pas, dans son essence, une approche de type résolution de problème où la réponse est donnée à partir d'une formulation claire de la question et de l'analyse de la situation. Le plus souvent, il s'agit au contraire de formuler des réponses en fonction de différentes hypothèses du futur anticipé, en fonction de ce que l'on sait et d'hypothèses sur ce que l'on ne sait pas -du moins pas encore-, finalement de s'appuyer sur des réponses incertaines et provisoires.

11 Le caractère intentionnel et souvent discursif, qui domine depuis les fictions de l'amont jusqu'aux choix essentiels, traduit, comme nous l'avons écrit plus haut, l'idée de projetvisée, véritable déclaration d'intention ou profession de foi, alors que le projetprogramme qui en est l'expression concrète se construit dans l'action et des productions successives et planifiées (Ardoino, 1987). En nous appuyant sur cette approche théorique et les paramètres d'analyse d'un projet proposés par Boutinet (1990) nous caractériserons les projets des étudiants au regard des dimensions suivantes: les intentions et buts explicités (insertion ou mobilité professionnelle, indépendance personnelle, visées alternatives, etc.) ; les motifs évoqués en matière de trajectoire antérieure (identification des ressources, opportunités et contraintes scolaires, personnelles, ou de carrière, etc.); les stratégies et moyens de formation préfigurés (acquisition de savoirs et méthodes, réalisation de stages, etc.).

Ainsi conçu, le projet professionnel et personnel de l'étudiant peut se présenter comme un investissement personnel que l'offre de formation -ensemble d'activités planifiées, coordonnées et maîtrisées- cherche à affiner, guider, adapter aux circonstances fortuites et à valoriser par ses réalisations et son but. En ce sens, le projet institutionnel de formation du Master peut être considéré comme le «creuset» du projet professionnel et personnel de chaque étudiant.

\section{La démarche de recherche}

Notre démarche de recherche est structurée par l'hypothèse de travail affirmant l'existence d'un projet professionnel et personnel évolutif et plus ou moins explicite, lié à la trajectoire antérieure de l'étudiant, dont le programme de formation vise à soutenir la rationalisation, la concrétisation et la valorisation. Nous avons donc mis en œuvre une enquête afin de recueillir et analyser des données sur les projets des étudiants de la promotion 2007-2008 lors de leur entrée en Master. Il convient de préciser que cette année là, les auteurs de l'article intervenaient comme enseignant dans le cadre de ce diplôme.

\subsection{L'échantillon d'enquête et le recueil des données par questionnaires}

L'échantillon visé se compose de vingt étudiants dont seize femmes et quatre hommes. Leur âge varie de 23 à 42 ans. Dans la promotion à laquelle nous nous intéressons, près de deux étudiants sur trois sont inscrits dans le cadre de leur parcours de formation initiale (précédant leur première insertion professionnelle) tandis que les autres étudiants sont inscrits au titre de la formation continue (développement de compétences en cours d'emploi, retour ou maintien dans l'emploi). 

trajectoire antérieure du stagiaire. Celle-ci est abordée tout d'abord à travers une dizaine de questions fermées portant sur des informations socio-démographiques ${ }^{3}$ (notamment le sexe, l'âge, les diplômes acquis et les expériences professionnelles significatives). Ensuite, trois questions ouvertes réclament les points de vue réflexifs des étudiants sur cette trajectoire. Il s'agissait pour ces derniers de réfléchir successivement à la contribution de certaines expériences scolaires, professionnelles puis personnelles ou socioculturelles à leur engagement en formation ${ }^{4}$. La seconde partie du questionnaire porte sur les caractéristiques du « projet » ambitionné en début de formation à travers trois questions ouvertes. La première question ${ }^{5}$ a pour but de détailler ce projet sur un plan professionnel en se déclinant sous la forme de quatre sous-thèmes : 1) le type d'insertion visée (première insertion, mobilité ou reconversion) et son aspect temporel (insertion immédiate ou différée) ; 2) le secteur professionnel ou le métier visé, ses intérêts et ses contraintes; 3) les savoirs et compétences que l'étudiant pense devoir acquérir; 4) l'existence d'un projet ou d'opportunités alternatifs. La seconde question ${ }^{6}$ porte sur la manière dont l'étudiant estime que la formation de Master va contribuer aux objectifs fixés. Cette question est composée de trois sous-thèmes: 1) l'apport des enseignements et des mises en situation professionnelle ; 2) la construction de réseaux socioprofessionnels ; 3) l'écriture du mémoire. Enfin, la troisième question ${ }^{7}$ demande aux étudiants de décrire les visées personnelles auxquelles leur engagement en formation répond sur les plans suivants : le développement de leur réseau de connaissances; le partage d'expériences collectives ; l'accroissement de leur autonomie ; l'anticipation d'évolutions en cours.

Notre corpus empirique est donc constitué par les réponses obtenues à ce questionnaire et retranscrites à l'aide d'un logiciel de traitement de texte. Ce corpus a été soumis à une démarche d'analyse de données textuelles assistée par le logiciel Alceste que nous exposons dans la section suivante. 


\subsection{L'analyse des données textuelles relatives aux projets par le logiciel Alceste}

19 Alceste. Ce choix méthodologique repose sur le fait que l'utilisation d'un tel logiciel entraîne une systématisation et une objectivation de l'analyse textuelle, qui tendent à réduire le poids des attributions préconçues du chercheur dans la définition des unités d'analyse, par rapport à un traitement manuel. Au moyen de procédures statistiques que nous détaillons plus loin, Alceste permet en effet de réaliser une réduction et un classement des segments d'un texte, "c'est-à-dire d'en donner un condensé parfaitement formalisé et d'en construire la structure " (Marchand, 1998, p. 52). Le travail interprétatif du chercheur visant à rendre compte des relations de sens établies par le ou les sujets est donc mené, dans un second temps, sur cette construction de la structure formelle du discours. Il convient cependant de souligner que les résultats quantitatifs produits par ces procédures statistiques doivent être considérés comme des indicateurs partiels et non comme des éléments de preuve d'une compréhension globale et absolue (Ibid.). Toutefois, l'utilisation du logiciel Alceste s'avère particulièrement intéressante pour accéder aux différents niveaux d'analyse de la structure textuelle (le lexique, les propositions et le texte en lui-même) (AubertLotarski \& Capdevielle-Mougnibas, 2002).

Sur le plan technique, ce logiciel réalise tout d'abord une analyse des caractéristiques lexicales 8 des corpus textuels. A partir d'une classification hiérarchique descendante, Alceste construit ensuite des classes de vocabulaire sur la base de la distribution et de la cooccurrence des mots qui composent les subdivisions du corpus (phrases et segments de phrases) retenues pour l'analyse (voir les résultats ci-dessous). Cette analyse statistique distributionnelle est basée sur le calcul du Chi2 appliqué aux mots et aux groupes de mots. Indépendamment du sens, les classes de discours obtenues regroupent donc des mots qui leur sont significativement rattachés, issus des réponses au questionnaire d'un ou plusieurs sujets réels et qui présentent des «environnements» de cooccurrence comparables (Marchand, 1998). Enfin, non seulement le chercheur peut décrire ces classes à partir de leur vocabulaire caractéristique, mais il peut enrichir et affermir sa compréhension des discours en tenant compte du contexte dans lequel ce vocabulaire est utilisé. En effet, le logiciel permet d'accéder aisément aux phrases (ou aux parties de phrase) qui contiennent le lexique spécifique de chaque classe.

21

En outre, le logiciel Alceste procède à une analyse factorielle des correspondances sur les résultats de la classification hiérarchique descendante (voir les résultats ci-après). A partir d'un tableau indiquant en colonne les classes et en ligne les différentes formes analysées, ce traitement statistique permet de rendre compte des rapports de proximité, d'indépendance et d'éloignement entre les différentes parties du corpus dégagées par la classification hiérarchique (Marchand, 1998). En fonction du nombre de facteurs extraits ${ }^{9}$ par cette procédure statistique, le chercheur obtient différents graphiques à deux dimensions dans lesquels les positions des classes et de leur lexique varient selon leur lien avec les facteurs (une position extrême d'une modalité indique une forte «corrélation " avec un facteur). Ainsi, en décrivant les concordances et les oppositions que soulignent les modalités particulièrement liées à tel ou tel facteur, il 
est possible de caractériser les lignes de force qui structurent les réponses ouvertes au questionnaire.

\subsection{Les résultats du traitement des données}

La classification des réponses aux questionnements ouverts conduits en début de Master aboutit à la construction de huit classes de discours (voir la figure 1 ci-dessous). L'analyse de ce traitement statistique montre que la classe 3 constitue la première classe apparue de façon stable. La classification obtenue se caractérise également par deux ensembles de classes emboîtées : d'une part, les classes 2, 6 et 8 et, d'autre part, les classes $1,4,5$ et 7 . Cette partition emboitée souligne que les classes appartenant à chacun des deux ensembles (les classes 2, 6 et 8 d'un côté et les classes 1, 4, 5 et 7 de l'autre) comportent des profils lexicaux proches.

Figure 1. Classification hiérarchique des réponses au questionnaire et poids ${ }^{10}$ des classes et regroupements de classes obtenus

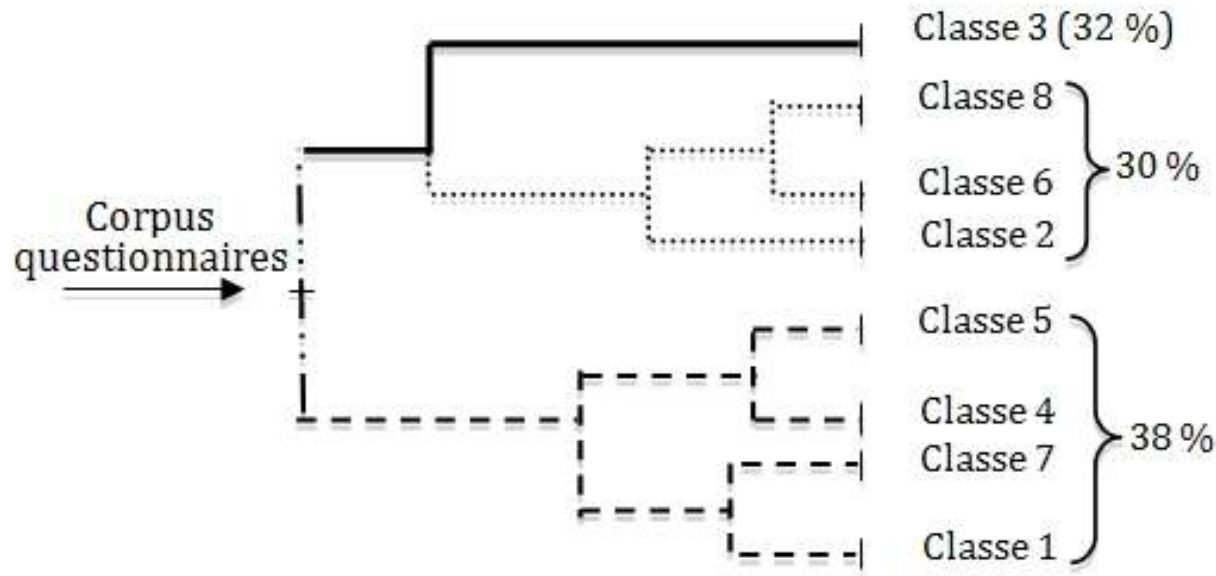

Parmi l'ensemble des résultats produits par la classification hiérarchique des réponses au questionnaire, nous nous sommes plus particulièrement basés sur le vocabulaire et les phrases propres à chaque classe. Nous avons conduit notre travail interprétatif à partir des éléments relatifs aux projets statistiquement saillants et pertinents au regard de notre cadre théorique de référence. Cette sélection du lexique et des phrases caractéristiques de chaque classe est présentée dans les annexes de l'article. Notre développement présenté dans la section 4 est également étayé par des citations de réponses d'étudiants que nous avons choisies parmi les ensembles de phrases spécifiques de chaque classe de discours.

L'analyse factorielle des correspondances opérée par le logiciel Alceste conduit à l'extraction de sept facteurs "résumant» la totalité des résultats obtenus par la classification hiérarchique descendante. Chaque facteur résume une partie de l'information totale constituée par ces résultats, exprimée en pourcentage ${ }^{11}$. Afin de favoriser l'intelligibilité de nos interprétations dans les limites imposées par cet article, nous ne prendrons en compte que les deux premiers facteurs extraits par l'analyse factorielle. En effet, ces facteurs 1 et 2 résument à eux deux près de $54 \%$ des résultats de la classification abordés précédemment. 
La figure 2 ci-dessous est une représentation graphique simplifiée du plan factoriel opposant ces deux premiers facteurs. Les positions des classes y sont indiquées par les chiffres en gras. Les mots situés dans ce graphique sont à la fois pertinents pour nos interprétations et significativement corrélés avec l'un des deux facteurs ou avec les deux. Cette sélection a été réalisée à partir de la projection graphique produite par le logiciel Alceste et du tableau de corrélations des classes et du lexique avec les facteurs. Les ensembles délimités par les tirets regroupent le vocabulaire spécifique de chaque classe et groupes de classes. Seul le regroupement chevauchant la partie gauche de l'axe horizontal (facteur 1) n'est pas lié à une classe de discours.

Figure 2. Représentation simplifiée du premier plan factoriel

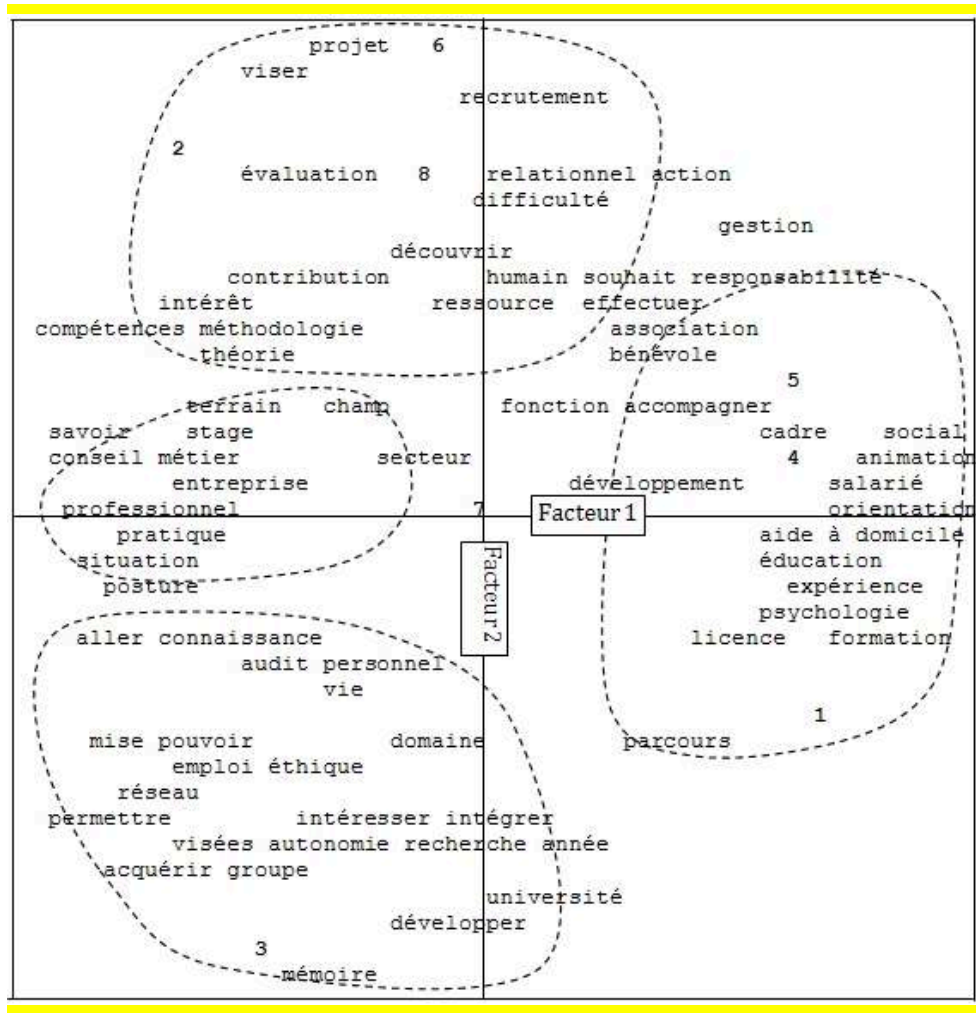

Nous interpréterons ces résultats en prenant principalement en compte les classes et les mots les plus représentatifs des deux facteurs considérés. Ceci nous conduira à rendre compte des principes de clivage entre les constituants majeurs des projets des étudiants dans la section 5 de cet article.

\section{Les composants essentiels des projets des étudiants}

Cette quatrième partie de l'article a pour but de caractériser la composition des projets des étudiants à partir d'une interprétation des résultats de la classification hiérarchique des réponses obtenues aux questions ouvertes de notre questionnaire. Comme nous l'avons vu dans la section 3.3., ces résultats distinguent la classe 3, l'ensemble des classes 2, 6 et 8 et enfin un regroupement des classes 1, 4, 5 et 7. Dans le but de présenter l'analyse la plus contrastée de ces résultats, nous présentons donc successivement la classe 3 puis globalement chacun de ces deux ensembles de classes. 
Cette interprétation en trois temps correspond, en effet, aux trois thèmes nettement hiérarchisés par la classification en lien avec notre approche conceptuelle du projet et son opérationnalisation dans notre questionnaire : la visée personnelle tout d'abord (classe 3), les attentes au regard de la formation qui s'engage (ensemble des classes 2, 6 et 8) et la mobilisation de l'expérience qui constitue, en fond, un matériau très abondant (ensemble des classes $1,4,5$ et 7 ). Ces éléments de motivation et d'engagement dans la formation dépendent des projets d'études antérieurs, de conditions de vie actuelles, du contexte universitaire et de la perception de l'insertion professionnelle, comme nombre de travaux l'ont montré (Romainville, 2000).

\subsection{En premier, des visées personnelles ... très sociales (classe 3)}

Point le plus significatif au plan statistique -autrement dit, classe des éléments de discours les plus saillants-, la thématique des visées personnelles s'organise autour des valeurs de l'apprentissage et du partage au sein du groupe de formation, de la réflexion et de la prise de recul sur les questions de développement professionnel et personnel. Ce « monde lexical » de la classe 3 regroupe $32 \%$ de l'ensemble des énoncés retenus par le logiciel Alceste dans son traitement statistique du corpus de questionnaires (voir la figure 1 ci-dessus).

Comme le montre les résultats présentés en annexe (voir le vocabulaire et les phrases spécifiques de la classe 3) il s'agit, selon les réponses, d'acquérir des connaissances dans le domaine du conseil et de l'accompagnement, de "partager des bouts de vie et d'expérience pour étoffer son parcours personnel et professionnel », de "tisser »un réseau de connaissances, de «questionner ses positionnements» et de "développer [sa] posture professionnelle ». Mais il s'agit également d'«apprendre à travailler en groupe », de " profiter de la dynamique de groupe et de la stimulation intellectuelle", de "gagner en assurance personnelle, en confiance et en autonomie ", et même de "mieux maîtriser le cours de [sa] vie " et de "[s]'épanouir professionnellement » en "apprenant des expériences des autres».

Bien que les discours portent sur les visées personnelles, la conception de la formation qui prévaut -sans être fermement énoncée- part du principe que compétences et savoirs s'acquièrent dans des pratiques tout à la fois collectives, plurielles et sociales. Semblablement, maints travaux en psychologie sociale ont confirmé que «les représentations sociales interviennent dans des processus aussi variés que la diffusion et l'assimilation des connaissances, le développement individuel et collectif des identités personnelles et sociales, l'expression des groupes » (Jodelet, 1989, p. 36-37).

31 Nous retiendrons cette idée que toute réalité se révèle affective, et donc tout projet immanquablement à la conjonction du subjectif et de l'objectif. Et comme le remarque Schneuwly (1987, p. 12) la formation s'inscrit dans un "processus d'appropriation d'expériences sociales, préexistantes, cristallisées sous de multiples formes dans des systèmes d'outils, d'objets produits et de signes ». Ainsi, en début de formation, les identités personnelles et identités professionnelles semblent-elles se confondre dans les projets des étudiants, sorte d'idéal fusionnel, de savoir apprendre ensemble, appelant à situer la formation dans des contextes diversifiés de communication, de collaboration et de coopération. 


\subsection{En second, des attentes plurielles à l'égard de la formation (classes 2,6 et 8 )} nombreux apports théoriques, au développement de compétences, à des interventions diverses et, surtout, au stage dans une organisation. Ces éléments répartis en trois classes (ici 2, 6 et 8 ) regroupent $30 \%$ des subdivisions du corpus traité par le logiciel (voir la figure 1 ci-dessus).

\section{l'évaluation, le conseil et l'accompagnement, mais aussi des connaissances en matière} d'ingénierie de formation, de "l'observation et l'analyse des situations de travail», de " l'analyse des pratiques professionnelles». Le souci porte aussi sur les apports méthodologiques divers développés en matière de conseil et d'accompagnement, et principalement des techniques tournées vers les individus comme la «conduite d'entretien de recrutement ou d'orientation ». L'attente générale renvoie donc à des savoirs fondamentaux liés à des disciplines spécifiques et à des savoirs de référence qui, s'appuyant sur des théories particulières, légitiment les pratiques professionnelles (voir les mots et phrases spécifiques des classes 2, 6 et 8 en annexe).

techniques attendues, citons encore les techniques de diagnostic, d'audit et de conseil, de gestion "des ressources humaines» et de résolution "des conflits", de coordination d'équipes. Sont aussi précisées les techniques d'écriture professionnelle, d'élaboration de rapports de bilans et de projets. Sur ce point, le mémoire professionnel est souvent évoqué comme un exercice extrêmement formateur et difficile, perçu en quelque sorte comme un chef-d'œuvre, au sens artisanal du terme. Il s'agit là de savoirs de base souvent considérés comme acquis, mais qui dépassent la simple maîtrise de techniques. Apprentissages et compétences collectifs sont là aussi rappelés fréquemment, évoquant en fond un climat d'apprenance collective (Carré, 2005).

La confrontation à la réalité des métiers du conseil, à la difficulté de contextes imaginés ou de situations imprévues, d'alternance ou de stage en cabinet de consultants évoque le réel souci d'" acquérir une posture professionnelle »et l'idée d'une professionnalisation entendue comme l'accès à "une position professionnelle supérieure » et "autonome ». La question des valeurs et de l'éthique est reprise et, sur ce point, on note que beaucoup d'étudiants perçoivent qu'un enseignement universitaire -à vocation critique- fût-il inscrit dans une logique de professionnalisation, ne correspond pas toujours aux pratiques réelles. D'où des d'interrogations sur une éthique contextualisée, des choix éthiques difficiles (recrutement, gestion des ressources humaines): "l'éthique professionnelle devient une dimension indispensable de la professionnalité. En interrogeant la place des valeurs dans l'activité, on réfléchit aux contours d'une pratique professionnelle, à ses possibilités tout autant qu'à ses limites " (Jorro, 2007, p. 20).

Les champs professionnels souhaités vont de la formation au travail social en passant par l'animation et les activités de service aux entreprises en matière de conseil en ressources humaines, de reclassement, d'audit. Les cabinets de consultants, les entreprises, les collectivités territoriales, les associations sont cités comme autant de possibilités d'insertion professionnelle. Le projet d'insertion marque encore plus fortement l'orientation des activités souhaitées vers les organisations: expertiseconseil auprès des institutions, ingénierie de projet, ingénierie pour des dispositifs

Revue internationale de pédagogie de l'enseignement supérieur, 26(1) | 2010 
d'évaluation, de formation, d'accompagnement, de développement des associations, management de projet et des organisations.

Si l'idée d'un projet alternatif est suscitée à l'évocation du caractère pluridisciplinaire de la formation, elle renvoie généralement aux mondes sociaux de la formation. Caractère prégnant, l'expectation, et plus subtilement l'expectative, décrit l'attente fondée sur des promesses, des probabilités, l'espérance d'un futur statut, mais aussi l'attitude prudente qui consiste à attendre pour se décider, pour agir utilement.

\subsection{En fond, la mobilisation des acquis de l'expérience (classes 1, 4, 5 et 7 )}

Cet ensemble discursif majeur qui représente $38 \%$ des énoncés du corpus retenus dans l'analyse par le logiciel (classes 1, 4, 5 et 7) décrit ce que nos sujets considèrent comme leurs moyens propres, leur investissement de départ dans leur projet en quelque sorte. En l'occurrence, mobiliser son expérience, c'est mobiliser des savoirs particuliers, difficiles à discerner, à dissocier des activités, incorporés, produits dans et pour l'action et à résonance fortement identitaire (Wittorski, 2007). Mais si cette expérience est perçue comme une construction fondamentalement personnelle, source d'un indéniable sentiment de compétence, les discours des étudiants soulignent là aussi que l'appropriation et l'actualisation des savoirs et des compétences s'inscrivent dans une dynamique sociale et collaborative.

La reconnaissance de la dimension formatrice de l'expérience s'appuie tout d'abord sur les expériences de formation. La majorité des étudiants engagés dans le Master professionnel viennent y parachever leur formation initiale, ayant pour la plupart des cursus en sciences humaines et sociales, avec des mentions en formation, animation ou développement social. Des études en sciences économiques sont aussi signalées, rappelant les savoirs acquis notamment en droit, gestion ou management. Moins fréquents sont les parcours pluridisciplinaires et la diversité s'observe surtout parmi les salariés recrutés dans le Master qui ont en commun d'avoir alterné des contrats de travail et des formations spécifiques à la formation de formateurs, l'éducation spécialisée ou l'animation, mais aussi au droit du travail, au commerce ou à l'environnement. L'autodidaxie n'est mentionnée qu'une seule fois, soulignant le besoin d'un soutien social, d'une reconnaissance professionnelle et l'occasion de se projeter dans une carrière.

L'expérience du travail, confirmée ou occasionnelle, est souvent soulignée comme source d'attrait pour les métiers du conseil et de l'accompagnement (voir en annexe les phrases significatives des classes 1, 4, 5 et 7). C'est au cours d'un emploi saisonnier qu'un tel a découvert son intérêt pour la gestion du personnel, au cours de stages que d'autres ont observé ou participé à des activités d'information, d'orientation voire de recrutement: «Pendant deux mois, j'ai pu travailler dans le secteur des ressources humaines (...) en tant qu'agent administratif vacataire (...). Ainsi, je suis rentrée en relation avec le personnel de ce service et j'ai développé un grand intérêt pour les ressources humaines ». Tous les salariés, inscrits au titre de la formation continue, considèrent cette formation comme une suite logique à leurs activités antérieures, en continuité de leur trajectoire et leurs acquis professionnels. Certains disent avoir déjà été repérés comme « personnes ressources " en matière de conseil dans leur environnement professionnel, ce qui les a confortés dans leur départ en formation. Le Master est alors généralement perçu 
comme une occasion de changement de fonction et de statut, un moment au service d'un projet de reconversion et d'une mobilité professionnelle balisée par l'expérience acquise. Le réalisme de telles aspirations rend décisives les réponses de la formation aux attentes de résultat.

Le point de vue sur l'apport des expériences professionnelles est d'autant plus tranché que les postes ou fonctions occupés sont fortement valorisés : dirigeant de société, senior manager, coach, auditeur, formateur de formateurs. La reconnaissance de la dimension formatrice du travail transparaît encore dans l'évocation des métiers du travail social, de la formation ou de l'animation. Nombreux parmi les plus jeunes décrivent les acquis tirés d'activités menées dans le cadre d'emplois à durée limitée (par exemple, assistant d'éducation dans un établissement scolaire), dans la conduite de projets et la gestion d'équipes, pointant systématiquement les transferts de savoirs et les enrichissements interactifs générés dans les situations vécues. L'importance du facteur relationnel est sans cesse rappelée, estompant souvent la difficulté de situations de travail ou le poids manifeste des contraintes décrites.

Carrières objectives car déjà réalisées, ou subjectives car projetées, se répondent comme en écho dans l'évocation des projets professionnels tous personnalisés. Cette expérience génératrice de développement intègre aussi nombre d'expériences personnelles vécues hors formation ou hors travail. L'engagement «dans le milieu associatif " ou l'action socio-éducative "dans le domaine périscolaire » sont considérés comme « des sources essentielles d'expériences personnelles formatrices " par les uns, tandis que telle autre estime avoir développé "des capacités d'écoute et de conseil auprès de la famille et d'amis ». Toute activité prend une valeur particulière dès lors qu'elle vient conforter le projet professionnel et personnel. Ainsi, la "création d'une association de jeunes", le "développement d'une association culturelle et sportive» ou "l'animation de réunions» associatives marquent-elles le souci d'une efficacité certaine. Le soutien scolaire, la gestion d'une bibliothèque ou la pratique de la création artistique collective traduisent on ne peut mieux l'idée de solidarité. L'accompagnement de "séjours de vacances adaptés pour personnes en difficulté ", "le bénévolat » ou la "coordination d'actions d'animation socioculturelle » éclairent l'idéal de partage ici associé aux activités de conseil et d'accompagnement.

43 Toutes ces pratiques bénévoles citées ont forgé une réelle conviction d'agir utilement, pour autrui avant tout, pour éprouver des talents naissants ou confirmés également. Ce sentiment d'aide, d'utilité sociale, d'un dû comme épreuve de soi explicite les valeurs de solidarité, de partage, d'efficacité et pose les bases éthiques de l'action altruiste. De l'éthique en actes en quelque sorte, dans lesquels s'ancre le projet professionnel et personnel.

\section{Des projets en tension, entre engagement et intégration}

Cette partie de l'article vise à mettre en évidence les rapports d'éloignement et de proximité qu'entretiennent, dans les discours, les éléments constitutifs des projets étudiants détaillés dans la section précédente. Pour ce faire, nous nous sommes intéressés aux résultats de l'analyse factorielle opérée par le logiciel sur la classification des réponses au questionnaire (facteurs 1 et 2). Nous avons mené notre travail interprétatif à partir de la projection factorielle du lexique spécifique de chaque classe 
et groupes de classes que nous avons circonscrite en quatre ensembles distincts (voir la figure 2). La figure 3 ci-dessous synthétise les oppositions entre ces quatre regroupements. Chaque ensemble a été désigné en fonction du sens du vocabulaire qui le compose, que nous avons interprété en relation avec notre approche conceptuelle du projet.

Figure 3. Les oppositions entre les constituants majeurs des projets des étudiants

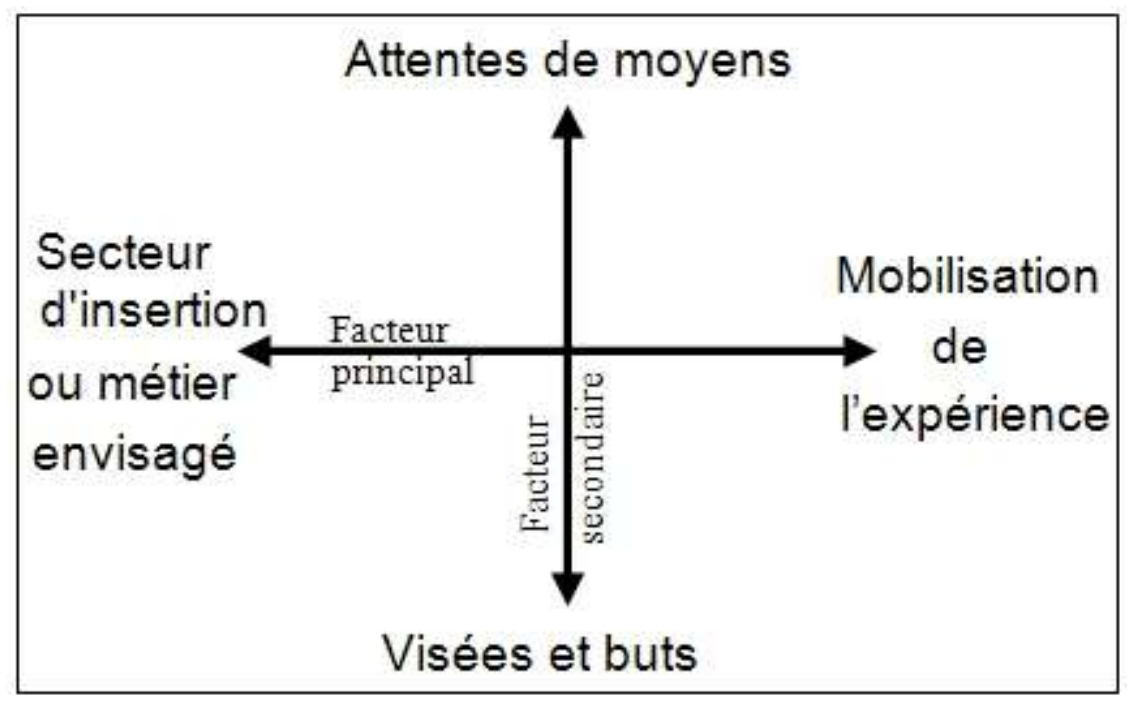

Le facteur principal (facteur 1) oppose le long récapitulatif des expériences mobilisées en vue de la formation de Master aux éléments de discours explicitant le métier ou le secteur professionnel visé et ses motifs. En toute logique, l'évocation de cette insertion est fortement liée au lexique sur la mise en situation professionnelle qu'autorise la réalisation du stage en cours de Master (voir figure 2). C'est pourquoi cet ensemble lexical relatif au champ professionnel ambitionné provient de la décomposition partielle du regroupement des classes 2, 6 et 8 portant sur les attentes à l'égard de la formation et plus particulièrement lié au facteur secondaire. L'importance quantitative du facteur principal (plus du tiers des résultats de la classification résumés) souligne la portée de cette opposition entre l'insertion envisagée et l'expérience rassemblée. Cette opposition est sous-tendue par le fait que les références à ces deux univers lexicaux sont à la fois fréquentes et distinctes dans les corpus textuels. Ce résultat nous semble rendre compte d'une tension caractéristique des projets personnels et professionnels : ceux-ci ne surgissent pas ex nihilo, mais se construisent comme des alternatives possibles à des situations faites de contraintes et d'opportunités, les représentations d'un futur souhaité s'élaborant invariablement vis-à-vis des acquis du passé. Cette tension traduit aussi l'espoir d'un parcours possiblement maîtrisé qui s'entrevoit en miroir d'une trajectoire trop souvent imposée, ou du moins perçue comme telle. Rappelant le caractère opératoire de l'anticipation, une telle structuration montre que le projet de formation s'énonce chez nos étudiants comme un espace-temps, une transition où s'opère un changement personnel dont la représentation, fût-elle imprécise, affirme le sens comme signification et direction prise. Bref, un engagement irrémédiable.

Le second facteur oppose les visées personnelles aux attentes en matière de formation. Ces deux composantes des projets sont donc évoquées significativement dans des 
portions spécifiques du corpus discursif. On peut tout d'abord y lire la tension entre buts et moyens propre à tout projet, les moyens rassemblant ici les apprentissages escomptés en regard du dispositif de formation tel qu'il est représenté en début d'année (voir la figure 2). Il faut surtout y lire le poids des attentes dont le projet dans son entier est tributaire, facteur d'espoir quant aux apports de la formation, source d'inquiétude quant aux chances de succès. Quel que soit le scénario évoqué, la contribution de la formation en termes de compétences est jugée déterminante, et il est acquis qu'un professionnel compétent est avant tout expert en son domaine. L'analyse des projets des étudiants souligne par conséquent la singularité et l'interdépendance des visées et ressources escomptées, marquées par des intérêts et des activités notamment de conseil- préférentiellement orientées vers les individus ou bien les organisations. En somme, notre sujet façonne son projet individuel de formation en regard d'un programme institutionnel de formation compris à l'aune de ses propres buts et plus encore de ses attentes.

\section{Formations universitaires « à projets » et perspective de professionnalisation des étudiants} composition des trois familles discursives relatives à l'organisation « en projet » d'une vision générale partagée (et manifestement confortée) qui articule les éléments d'une visée, les représentations d'un programme et les moyens personnels acquis de l'expérience. L'interprétation de l'analyse factorielle nous a conduits ensuite à caractériser deux tensions qui condensent l'activité de projet telle qu'elle est décrite en début de formation entre insertion professionnelle envisagée et expérience d'une part, moyens et buts d'autre part.

A l'évidence, si l'enjeu des projets professionnels et personnels tient au pari des apprentissages nécessaires à l'exercice des métiers dans leur diversité, la trame de ces derniers se présente comme un enchaînement raisonné de prises de risque raisonnables et constitue un vecteur essentiel au développement professionnel. Plus encore, ces projets guident le développement professionnel en s'actualisant dans le projet institutionnel de formation tel que chaque étudiant se le représente. Mais, audelà de la mise en œuvre généralisée et "procédurale" d'une démarche de type " projet personnel et professionnel » dans le supérieur, quels enseignements tirer de ces résultats pour que l'organisation pédagogique du Master concoure au mieux aux projets des étudiants et, par-là même, à leur professionnalisation ?

Notre recherche confirme tout d'abord des résultats obtenus dans d'autres niveaux d'enseignement supérieur (par exemple, Beckers, 2007). Comme le montre le poids des références discursives aux pairs et à la collaboration dans les trois ensembles lexicaux, la construction des projets individuels tire parti du ressort de développement que composent les interactions sociales au sein du groupe de formation. Dès leur émergence à l'entrée en formation, les projets se construisent et se partagent chemin faisant, sollicitant de façon soutenue l'intercompréhension et contribuant de facto à la solidarité et la création de valeurs communes au groupe d'étudiants. On peut penser que l'importance de l'apport du collectif est à la mesure de la complexité de l'organisation et de l'économie du projet individuel, alors même que ces étudiants ont un niveau d'étude élevé et qu'ils ont réfléchi à leurs perspectives professionnelles en

Revue internationale de pédagogie de l'enseignement supérieur, 26(1) | 2010 
amont de leur engagement en formation. L'impétrant prend en effet tous les risques, tout à la fois maître d'ouvrage, bénéficiaire du résultat futur, maitre d'œuvre, architecte, ensemblier et responsable de la réalisation des tâches élémentaires. Ainsi, ces éléments tendent à nuancer les discours incitatifs à l'individualisation des parcours et de l'accompagnement: la mise en œuvre d'activités pédagogiques de suivi des étudiants sur un mode collectif et collaboratif semble au contraire requise pour permettre l'affermissement, l'évaluation et l'enrichissement croisés des projets "personnels». Plus généralement, le développement des dynamiques individuelles insérées dans une aventure collective et un ensemble encadré, stable, de procédures formatrices garantes de leur évolution devrait renforcer la pertinence et la robustesse des projets personnels et professionnels des étudiants.

50 Ces travaux soulignent par ailleurs la force des attentes de légitimation professionnelle que manifestent les projets des étudiants sur le plan des apprentissages, des techniques et de l'éthique. Si cette perspective appelle la conception d'un contenu de formation approprié aux exigences des métiers (savoirs et apports méthodologiques de référence), elle nécessite également de soutenir les étudiants dans la construction d'un projet en phase avec des enjeux professionnels authentiques et contextualisés (Bédard \& Béchard, 2009). Cela paraît plus nécessaire encore pour les formations à des métiers émergents, tel que le Master considéré dans cet article, qui conduisent à l'obtention de diplômes d'une certaine lisibilité pour les champs professionnels au point de vue des modalités de construction et de mise en œuvre de la formation mais qui ne délivrent pas un titre requis pour accéder à l'exercice de professions réglementées (par exemple, architectes, médecins...).

51 Le programme d'étude doit donc donner aux étudiants le temps et les moyens d'explorer en début de formation, différents terrains d'insertion possibles (recherches documentaires, rencontres et entretiens exploratoires avec des "gens du métier ») en fonction d'une problématique ou d'un questionnement d'ordre professionnel, tout en circonscrivant les champs de pratique pertinents. L'ancrage et la confrontation des projets aux réalités professionnelles doit en outre conduire les étudiants à mieux identifier les compétences et les postures pertinentes à maitriser et à mobiliser dans les métiers visés. A court terme, ce volet permet de clarifier et d'améliorer l'articulation de ces contraintes à leur projet, au choix de leur terrain de stage et de la problématique professionnelle qu'ils exposeront dans leur mémoire de fin d'étude. Cet enjeu de formation peut sembler à priori moins pertinent pour des étudiants inscrits en formation continue dans le cadre de leur emploi et ayant par conséquent une expérience concrète des champs professionnels. Pour ces derniers, une véritable visée de formation professionnalisante ${ }^{12}$ impliquerait plutôt de les amener à construire un projet personnel et professionnel émancipé sinon distancié, des projets et problématiques spécifiques de leur lieu de travail habituel. Dans un même but de répondre aux attentes de légitimation de la valeur des projets, certains dispositifs pédagogiques spécifiques pourraient engendrer un gain de reconnaissance professionnelle non négligeable : par exemple une évaluation en milieu universitaire de la qualité des projets prenant en compte des critères du monde du travail, l'organisation de manifestations symboliques lors de la remise des rapports et travaux étudiants sur leurs terrains de stage.

52 Enfin, l'étude de nos matériaux empiriques montre le caractère prévalent de la trajectoire antérieure des étudiants comme vecteur de moyens à mobiliser dans leur 
projet. La sur-représentation des étudiants en formation initiale par rapport aux étudiants en formation continue dans l'échantillon d'enquête explique certainement la hiérarchisation de ces expériences que l'analyse statistique réalisée nous offre de manière nette: l'expérience académique est placée avant l'expérience professionnelle et l'expérience personnelle, autrement dit les savoirs transmis avant les savoirs d'action mis en valeur. Ces différents types de ressources sont néanmoins évoqués de manière conséquente dans les projets et les dispositifs pédagogiques semblent donc devoir soutenir tous les étudiants dans la mise à profit de leurs expériences de formation, de travail ou de bénévolat. Ce d'autant plus que la diversité de ces expériences et la complexité des combinaisons qu'elles génèrent vont être renforcées avec les étudiants amenés à s'engager dans des parcours universitaires (Bédard \& Béchard, 2009). Or, les processus de décentration des stagiaires concernant leur trajectoire antérieure ainsi que la conceptualisation, la formalisation et l'intégration des savoirs d'action procèdent notamment d'un travail d'écriture et de ré-écriture du projet (explicitation et discussion du projet, évaluation et bilan dans le mémoire professionnel) et d'une posture réflexive qui ne vont pas de soi pour tous les étudiants, même de second cycle universitaire. Plus encore, ce travail pourrait s'avérer à la fois plus précieux et plus difficile pour des étudiants ayant une expérience professionnelle riche mais éloignée des systèmes d'exigences académiques. Ainsi, des séquences pédagogiques consacrées aux discours de "présentation de soi» (Tralongo, 2008, p.101) ajustés aux modes d'écriture des projets dans les formations universitaires professionnalisantes (mise en lien et en cohérence des éléments de la trajectoire, récit " actif» et "distancié » des expériences, lexique de description des activités) pourraient être nécessaires pour ces étudiants. L'articulation de ce travail d'écriture du projet avec celui de conception d'un autre outil d'apprentissage intégrateur, le "portfolio professionnel étudiant», pourrait aussi développer la réflexion de ces derniers sur leurs expériences, leurs réalisations et leur cheminement (Hébert, Beaudoin, Thibaut \& Pitre, 2009).

\section{Limites de la recherche et conclusion}

L'examen des projets des étudiants d'un Master à leur entrée en formation nous a permis de rendre compte des représentations qui se déploient dans une tension entre expectation et expérience. Nous avons ainsi analysé leur agencement autour de visées personnelles et professionnelles fortement socialisées, d'attentes au regard des éléments constitutifs du programme d'étude et de moyens que leur propre expérience semble leur assurer. Il convient néanmoins de souligner les principales limites de cette recherche. Nous n'avons tout d'abord effectué qu'une seule collecte d'informations sur les projets à l'entrée en formation. Notre matériel empirique ne nous a donc pas permis pas de saisir les dynamiques individuelles et collectives d'évolution des projets en rapport avec le développement professionnel des étudiants, alors même que notre approche conceptuelle précise le caractère provisoire et incertain des projets.

Une seconde limite de cette recherche touche à la dimension interprétative de notre démarche. Si nous avons choisi d'utiliser le logiciel Alceste pour objectiver une partie de la procédure d'analyse textuelle, il n'en reste pas moins que la qualité de notre développement est soumise à la fiabilité des résultats produits par les traitements informatiques et à la validité de notre interprétation de ces résultats. Concernant les 
limites inhérentes aux procédures statistiques du logiciel, il faut rappeler que ces dernières produisent des indicateurs à l'intérêt heuristique certain, mais qui restent une formalisation particulière $\mathrm{du}$ corpus discursif qui suppose des opérations de classement et de réduction indépendantes du sens des mots. C'est pourquoi il est nécessaire de replacer systématiquement les formes constituant le vocabulaire des classes dans les phrases qui les contiennent. Concernant les limites propres au travail interprétatif, nous avons également cherché à réduire les effets de la subjectivité en confrontant les interprétations des deux chercheurs, en procédant à une analyse thématique du lexique des classes d'abord centrée sur les mots les plus significatifs au plan statistique, et par une connaissance préalable de l'ensemble du corpus de discours. Pour autant, il aurait été pertinent d'accroître la «validité de signifiance » de nos interprétations (Pourtois \& Desmet, 2007, p. 57) en les soumettant aux points de vue critiques des étudiants du Master, par exemple au cours d'entretiens complémentaires.

Enfin, une troisième limite de notre recherche porte sur l'échantillon d'enquête dont la taille réduite et les spécificités des sujets (sur-représentation des étudiantes, en formation initiale) restreignent la généralisation possible des résultats obtenus. Plus généralement, la composition des projets mises en évidence tient aussi à la place d'un cursus universitaire professionnalisant en sciences humaines au sein de l'enseignement supérieur français (caractérisé par la dualité entre universités et écoles professionnelles) qui joue notamment sur le type d'étudiants recrutés (linéarité du parcours de formation, niveau scolaire, moyens de financement des études, etc.). La généralisation de conclusions nécessiterait par conséquent de conduire des recherches comparables dans d'autres contextes français ou étrangers de formation ou une mise en perspective avec des travaux existants. Peu d'enquêtes se sont toutefois intéressées comme nous le faisons ici, aux projets des étudiants dans les formations de niveau supérieur aux premiers cycles universitaires.

56 Nous avons néanmoins cherché à mettre en évidence les implications pédagogiques qui découlent de la structuration des projets analysés (travail collectif des projets, reconnaissance professionnelle, formalisation de l'expérience). Nous avons par conséquent cerné quelques pistes organisationnelles pour que le projet professionnel et personnel et son étaiement par un dispositif pédagogique intégré deviennent réellement structurant pour les apprentissages en formation de haut niveau et contribuent ainsi au processus de professionnalisation des étudiants. L'intérêt de ces stratégies réside dans le fait qu'elles s'appuient moins sur un modèle pédagogique préalable que sur la mise au jour des composants des projets à partir d'un cadre théorique et d'une d'investigation empirique.

Mais, si « finalement, il n'est jamais de pédagogie sans projet » (Ardoino, 1987), la mise en œuvre d'un tel dispositif peut néanmoins soulever des questions liées au degré de connaissance des métiers en milieu universitaire ou à l'efficience des partenariats université-champs professionnels. Comment les enseignants-chercheurs peuvent-ils en effet assurer la pertinence, la faisabilité, l'authenticité et la contextualisation des projets de tous leurs étudiants alors même que se multiplient les formations professionnelles dans le système universitaire? Dans cette perspective, de telles «mutations » des pratiques enseignantes dans le supérieur (Rege Colet \& Romainville, 2006) pourraient cependant s'inspirer d'activités bien connues des universitaires, l'accompagnement et le suivi des projets... de recherche des étudiants. 
Nous remercions les deux experts anonymes dont les évaluations critiques et constructives ont contribué à l'amélioration de ce texte.

\section{BIBLIOGRAPHIE}

Ardoino, J. (1987). Finalement, il n'est, jamais de pédagogie sans projet. Éducation permanente, 87, 102-104.

Aubert-Lotarski, A. \& Capdevielle-Mougnibas, V. (2002). Dialogue méthodologique autour de l'utilisation du logiciel Alceste en sciences humaines. In Lexicometrica (Éd.), Actes des $6^{\text {ème }}$ Journées internationales d'Analyse statistique des Données Textuelles (pp. 45-56). Consulté le 10 octobre 2008 à : http://www.cavi.univ-paris3.fr/lexicometrica/jadt/jadt2002/tocJADT2002.htm.

Bart, D. (sous presse). Mise en situation professionnelle : enjeux et signes de reconnaissance entre stagiaires et référents. In A. Jorro \& J.-M. De Ketele (Éds.), La professionnalité émergente. Quelle reconnaissance? (Titre provisoire). Bruxelles : De Boeck.

Beckers, J. (2007). Compétences et identité professionnelles. L'enseignement et autres métiers de l'interaction humaine. Bruxelles : De Boeck.

Bédard, D. \& Béchard, J.-P. (2009). Quelques conditions pour un curriculum en développement au supérieur. In D. Bédard \& J.-P. Béchard (Éds.), Innover dans l'enseignement supérieur (pp. 249-266). Paris : PUF.

Beret, P. (2002). Projets professionnels et emplois ultérieurs : une analyse des mécanismes de l'insertion professionnelle. L'Orientation Scolaire et Professionnelle, 31(2), 179-194.

Boltanski, L. \& Chiapello, E. (1999). Le Nouvel Esprit du capitalisme. Paris : Gallimard.

Boutinet, J.-P. (1986). Le concept de projet et ses niveaux d'appréhension. Education permanente, $86,5-26$.

Boutinet, J.-P. (1990). Anthropologie du projet. Paris : PUF.

Carré, P. (2005). L'apprenance. Vers un nouveau rapport au savoir. Paris : Dunod.

Dewey, J. (1938). Experience and education. New-York : The Macmillan Company.

Hébert, M., Beaudoin, J.-P., Thibaut, R. \& Pitre, R.(2009). Conception, implantation et utilisation d'un portfolio professionnel étudiant dans un programme de formation en ergothérapie. Revue internationale de pédagogie de l'enseignement supérieur [En ligne], 25(1). Consulté le 01 novembre 2009 à : http://ripes.revues.org/index109.html

Jodelet, D. (1989). Les représentations sociales. Paris : PUF.

Jorro, A. (2007). L'évaluation, génératrice de développement professionnel ? In A. Jorro (Éd.), Evaluation et développement professionnel (pp. 11-31).Paris : L'Harmattan.

Marchand, P. (1998). L'analyse du discours assistée par ordinateur. Paris : Armand Colin.

Masclet, J. \& Leconte, C. (2007). Le projet personnel et professionnel : création d'une démarche fonctionnelle adaptée à l'IUT. Psychologie du travail et des organisations, 13(2), 73-94. 
Mérini, C. \& Séré, M.-G. (2001). Le module Projet professionnel : une ouverture en premier cycle universitaire, un équilibre dynamique à trouver. Revue française de pédagogie, 136, 21-29.

Pelletier, D., Noiseux, G. \&Bujold, C. (1974). Développement Vocationnel et Croissance Personnelle. Montréal : Mac Graw-Hill.

Pineau, G. \& Michèle, M. (1983). Produire sa vie. Autoformation et autobiographie. Montréal : SaintMartin.

Pourtois, J.-P. \& Desmet, H. (2007). Épistémologie et instrumentation en sciences humaines. Liège : Mardaga.

Rege Colet, N. \& Romainville, M. (Éds.) (2006). La pratique enseignante en mutation à l'université. Bruxelles : De Boeck.

Roegiers, X. (2003). Analyser une action d'éducation ou de formation. Bruxelles : De Boeck.

Romainville M. (2000). L'échec dans l'université de masse. Paris : L'Harmattan.

Schneuwly, B. (1987). Les capacités humaines sont des constructions sociales. Essai sur la théorie de Vygotski. Journal européen de psychologie de l'éducation, 1, 5-17.

Tralongo, S (2008). La méthode du « Projet personnel et professionnel » : un « travail de soi » des étudiants. Sociologie pratique, 2(17), 95-105.

Wittorski, R. (2007). Professionnalisation et développement professionnel. Paris : L'Harmattan.

\section{NOTES}

1. La première massification correspond en France à l'explosion des effectifs d'étudiants inscrits dans les universités au cours des années 1960.

2. Référence à l'origine latine momentum contraction de movimentum, mouvement.

3. En raison de la taille de notre échantillon et de la diversité des réponses relatives à ces questions d'ordre sociodémographique, les traitements statistiques (voir plus loin) n'ont pas permis de faire émerger significativement l'une ou l'autre des variables correspondantes. Ces dernières n'ont donc pas pu être véritablement utilisées dans l'analyse des données.

4. Ces trois questions ouvertes étaient formulées de la manière suivante: «A votre avis, certaines formations antérieures ont-elles particulièrement favorisé votre orientation vers le Master Conseil en développement professionnel?»; «A votre avis, certaines expériences professionnelles ont-elles significativement participé à votre orientation vers le Master 2 Conseil en développement professionnel ? ; « Selon vous, certaines expériences personnelles ou sociales ont-elles particulièrement contribué à votre orientation vers le Master 2 Conseil en développement professionnel?".

5. Cette question générique était formulée de la manière suivante : «Pouvez-vous préciser le projet professionnel en regard duquel vous vous engagez dans cette formation?».

6. Cette seconde question générique était énoncée ainsi: «Selon vous, en quoi le Master 2 «Conseil en développement professionnel» va-t-il contribuer au projet professionnel que vous venez de décrire?».

7. La troisième question de la seconde partie du questionnaire était formulée ainsi : «Le fait de s'engager dans cette formation répond également à des visées plus personnelles. Pouvez-vous les décrire?».

8. Alceste donne ainsi des indications sur la fréquence d'utilisation des noms, des adjectifs, des adverbes, des marqueurs d'une modalisation, des marqueurs d'une relation temporelle, etc. 
9. Les classes obtenues par la classification hiérarchique descendante et le lexique qui les composent sont considérés comme des variables dans l'analyse factorielle. Cet ensemble de variables peut être représenté par un nuage multidimensionnel de points, doté d'une inertie totale. Cette inertie rend compte de la dispersion du nuage de points par rapport au centre de gravité de ce nuage. L'analyse factorielle des correspondances consiste à définir successivement des facteurs qui sont des droites de régression « résumant " l'information relative aux positions spatiales des éléments (droites maximisant l'inertie extraite du nuage multidimensionnel). Le logiciel Alceste extrait des facteurs jusqu'à épuiser l'inertie totale.

10. Le poids en pourcentages des classes et regroupements de classes indique la proportion des énoncés retenus par le logiciel dans le traitement statistique du corpus que ces classes et groupes de classes rassemblent.

11. Les pourcentages d'information résumée par les facteurs sont: facteur $1=34 \%$, facteur $2=$ $20 \%$, facteur $3=16 \%$, facteur $4=11 \%$, facteur $5=8 \%$, facteur $6=7 \%$, facteur $7=4 \%$.

12. Dans la mesure où la professionnalisation des acteurs est définie comme un processus d'accroissement de leur maîtrise et de leur efficacité (individuelle et/ou collective) pour résoudre des problèmes complexes de manière autonome (Wittorski, 2007).

\section{RÉSUMÉS}

Cet article rend compte de la structuration des projets d'étudiants participant à une formation professionnalisante de second cycle universitaire (Master) et de la manière dont ces derniers «traduisent» le programme de formation en appui «stratégique» à leur développement professionnel. L'analyse des réponses de ces étudiants à une enquête par questionnaire permet de mieux comprendre les conditions d'émergence des projets et d'en dégager les différents agencements autour de trois composants majeurs: 1) les éléments d'une visée; 2) les représentations d'un programme de formation; et 3) les moyens personnels acquis de l'expérience. Nous montrons également en quoi ces projets soutiennent la pluralité des engagements et des apprentissages des étudiants. En conclusion, nous soulignons que cette recherche appelle des aménagements pédagogiques tenant compte de la configuration des projets mise au jour et de leur rôle de support du processus de professionnalisation.

This article analyzes projects carried out by students taking part in a professional academic master programme. It focuses on how the students "translate" the programme into "learning strategies" for their professional development. Analysing students' answers in a questionnaire enables to get a better understanding of the genesis of their projects and to bring out various issues regarding three main components : 1) elements of purpose, 2) conceptions regarding programmes and 3) skills acquired through experience. This article also shows in which ways these projects support a wide range of student commitments and learning strategies. To conclude, we emphasise that this research requires a pedagogical approach that takes into consideration the projects' design and their contribution towards professional development. 
INDEX

Keywords : professional development, professionalizing master's degree, project-based training, students projects

Mots-clés : formation à projets, formation universitaire professionnelle, master, professionnalisation, projet étudiant

\section{AUTEURS}

\section{DANIEL BART}

Maître de conférences en sciences de l'éducation

Université Lille 3 (France), équipe CIREL-Théodile

Adresse postale :

Université Charles-de-Gaulle - Lille 3, Domaine universitaire du "Pont de Bois", rue du Barreau BP 60149, 59653 Villeneuve d'Ascq Cedex

Courriel : daniel.bart@univ-lille3.fr

\section{MICHEL FOURNET}

Maître de conférences en sciences de l'éducation

Université Toulouse 2 (France), équipe CREFI.T

Adresse postale :

Université Toulouse 2, Maison de la Recherche, CREFI-T, 5 allées Antonio-Machado, 31058

Toulouse Cedex 9

Courriel : fournet@univ-tlse2.fr 